\title{
Gaussian models for the distribution of Brownian particles in tilted periodic potentials
}

\section{The limit of high barriers}

\author{
D.A. Kulikov ${ }^{1}$, N.V. Agudov ${ }^{1}$, and B. Spagnolo ${ }^{2, a}$ \\ 1 Radiophysics Department, Nizhni Novgorod State University, Nizhni Novgorod, Russia \\ 2 Dipartimento di Fisica, Group of Interdisciplinary Physics and CNISM, Università di Palermo, Viale delle Scienze pad. 18, \\ 90128 Palermo, Italy
}

Received 15 May 2011 / Received in final form 11 August 2011

Published online 23 September 2011 - (C) EDP Sciences, Società Italiana di Fisica, Springer-Verlag 2011

\begin{abstract}
We present two Gaussian approximations for the time-dependent probability density function (PDF) of an overdamped Brownian particle moving in a tilted periodic potential. We assume high potential barriers in comparison with the noise intensity. The accuracy of the proposed approximated expressions for the time-dependent PDF is checked with numerical simulations of the Langevin dynamics. We found a quite good agreement between theoretical and numerical results at all times.
\end{abstract}

\section{Introduction}

The model of the one-dimensional overdamped Brownian motion in tilted periodic structures appears in the analysis of different systems [1-5], like Josephson junction [6,7], phase-locked loop (PLL) systems $[8,9]$, protein transport, Brownian motors [10-12] and others. Specifically, the underdamped regime of the Brownian particle in a titled washboard potential at intermediate and low friction was investigated in references $[1,4,5]$. In reference [4], an excess diffusion of the travelling particle due to the trapped-torunning transition was revealed.

The main characteristic of the model under investigation is the non-stationary probability density function (PDF) of the Brownian particle coordinate, which has been subject of theoretical and numerical investigations $[2,10,13-15]$ and it is unknown up to now.

The asymptotic expression $(t \rightarrow \infty)$ of the time dependent PDF, for an arbitrary tilted periodic potential, was introduced in reference [14]. It consists of two factors: the Gaussian envelope and the intrawell distribution. The Gaussian envelope represents the coarse grained density of the Brownian particles, diffusing in the flat potential with constant tilt. While, intrawell distribution, together with the average drift and the effective diffusion coefficient, depend on the detailed structure of the periodical potential with barriers and wells.

Nevertheless, the exact non-stationary distribution is not obtained yet due to the mathematical difficulties, which are connected with the solution of the FokkerPlanck equation (FPE). In particular, in reference [15] a

\footnotetext{
a e-mail: bernardo.spagnolo@unipa.it
}

new approximated expression for the time-dependent PDF is introduced. There, it was shown that the proposed expression can well describe the time-dependent probability density function of overdamped Brownian particles, moving in tilted periodic potentials, for large and intermediate times. The parametric functions of the proposed expression of PDF were obtained self-consistently, through quite cumbersome calculations. This is not always useful, especially when one needs to get an analytical expression of other characteristics starting from the time-dependent PDF. This is the case of the autocorrelation function and the spectrum of the phase noise in PLL systems [16]. Therefore, simple approximate models for the PDF are also necessary.

In this paper we introduce two modelling functions (modelling distributions), describing the time dependent probability density of overdamped Brownian particles moving in a tilted periodic potential, in the limit of high potential barriers. These modelling distributions have a simple analytical expression, and are more useful for further theoretical calculations, estimations and analysis applied to specific problems. Simplification is obtained for the case of high potential barriers in comparison with the noise intensity. This case has been well studied for bistable and metastable systems $[1,17,18]$. Similar approaches can be used for tilted periodical potentials. Moreover, the limit of high potential barriers is often one of the most practical cases which describes the main running regimes of various systems (e.g. lock-in regime of PLL, duffusion in solids, etc.). The proposed model distributions are shown to have a good agreement with the results of numerical simulations of the Langevin dynamics. 


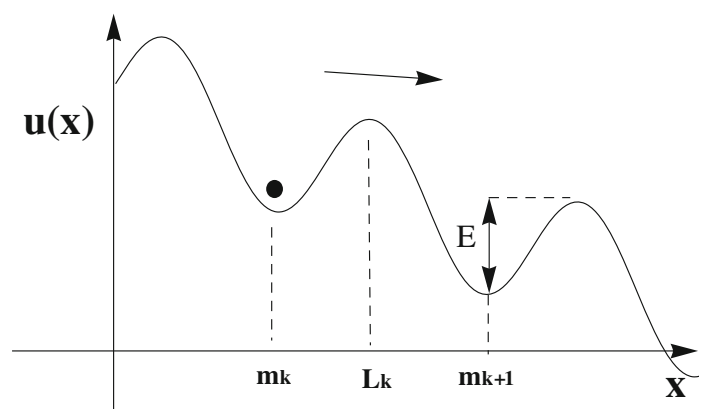

Fig. 1. Tilted periodic potential profile.

\section{Overdamped Brownian motion and asymptotic distribution}

The physical system considered is an overdamped onedimensional Brownian particle moving in a tilted periodic potential, and whose dynamics is described by the following Langevin equation

$$
\frac{d x}{d t}=a-F(x)+\xi(t), \quad x(0)=x_{0},
$$

where $x(t)$ is the Brownian particle coordinate, $a$ is an homogeneous static force, causing drift of particles, $F(x)$ is a periodic function $(F(x)=F(x+2 \pi)), \xi(t)$ is a randomly fluctuating force, that is a Gaussian white noise with zero mean $\langle\xi(t)\rangle=0$, and correlation $\langle\xi(t) \xi(t+\tau)\rangle=2 D \delta(\tau)$, with $D$ the noise intensity. The titled periodic potential $u(x)$ is

$$
u(x)=-a x+\int_{0}^{x} F\left(x^{\prime}\right) d x^{\prime} .
$$

Such potential is shown in Figure 1. Further we assume that the minima of the potential profile are situated in points $m_{i}$, where $i= \pm 1, \pm 2, \ldots$ and $2 \pi(i-1)<m_{i}<2 \pi i$.

In the absence of fluctuations $(\xi(t)=0)$, the particle will stay at the local potential minimum forever. In the presence of the random force $\xi(t) \neq 0$ and $D>0$, the particle can overcome the potential barriers and, due to the drifting force, moves in average to the right.

The Fokker-Planck equation (FPE) for the probability density function of the Brownian particle, corresponding to the Langevin equation (1), is $[1,19]$

$$
\frac{\partial W(x, t)}{\partial t}=\frac{\partial}{\partial x}\left(\frac{d u}{d x}+D \frac{\partial}{\partial x}\right) W(x, t),
$$

with initial condition $W(x, 0)=\delta\left(x-x_{0}\right)$ and boundary conditions $W( \pm \infty, t)=0$. This probability distribution $W(x, t)$ contains full information about Markov proccess $x(t)$. But up to now the non-stationary solution of the FPE for any periodic function $F(x)$ is not known. The timedependent PDF, for large times $(t \rightarrow \infty)$, tends asymptotically to $[2,14,15]$

$$
W_{a s}(x, t)=W_{s t}(x) \frac{e^{-\frac{\left(x-x_{0}-\nu t\right)^{2}}{4 D_{e f f}}}}{\sqrt{4 \pi D_{e f f} t}},
$$

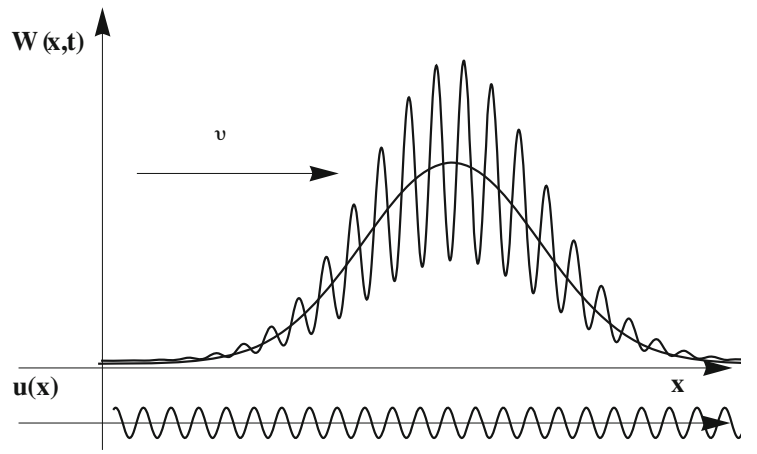

Fig. 2. The asymptotic PDF of equation (4). The thick line is the Gaussian coarse grained envelope. The potential profile without the tilt force is shown in the bottom of the figure.

where the average drift $\nu$ and the effective diffusion coefficient $D_{\text {eff }}$ are defined as follows

$$
\begin{gathered}
\nu=\left\langle\frac{d x}{d t}\right\rangle=\lim _{t \rightarrow \infty} \frac{\left\langle x(t)-x_{0}\right\rangle}{t}, \\
D_{\text {eff }}=\frac{1}{2} \lim _{t \rightarrow \infty} \frac{[x(t)-\langle x(t)\rangle]^{2}}{t} .
\end{gathered}
$$

Equation (4) consists of two factors. The Gaussian factor and the function $W_{s t}(x)$, which is the periodically continued stationary distribution with special normalization $2 \pi$

$$
\int_{2 \pi(k-1)}^{2 \pi k} W_{s t}(x) d x=2 \pi .
$$

The distribution $W_{s t}(x)$ is the stationary solution of the FPE (3) with periodic boundary conditions: $W_{s t}(x)=$ $W_{s t}(x+2 \pi)$. For an arbitrary tilted periodic potential profile (2), the stationary distribution is known and has the following expression $[1,13,20]$

$$
W_{s t}(x)=c e^{-u(x) / D} \int_{x}^{x+2 \pi} e^{u(z) / D} d z,
$$

where $c$ is a normalization factor.

Thus, in the limit of $t \rightarrow \infty$, the distribution $W(x, t)$ has the Gaussian coarse grained envelope, spreading and moving in the direction of the potential profile slope with the drift velocity $\nu$. The distribution $W_{s t}(x)$ defines the local structure, with minima and maxima of the PDF, corresponding to the maxima and minima of the periodic potential profile (see Fig. 2).

The exact analytical expressions for the average drift velocity and the effective diffusion coefficient are known for an arbitrary function $F(x)[2,10,14,21]$. Nevertheless, the expression (4) does not describe correctly the real distribution for short times. This was shown in reference [15], by comparing the PDF calculated form equation (4) with that obtained from numerical simulations of the Langevin equation (1) with a harmonic function $F(x)$. 
In this work we aim to get the model expressions for the time-dependent PDF, in the case of high potential barriers $E / D \gg 1$. Therefore, we can use appropriate expressions for $\nu$ and $D_{\text {eff }}$ obtained for the same case. In particular, we consider the harmonic function

$$
F(x)=R \sin x
$$

with the coordinate of the first minimum of the potential profile at $x_{1}=\arcsin (a / R)$. Therefore, the expressions for the mean drift velocity and the effective diffusion coefficient are (see $[21,22])$

$$
\nu=D \frac{\sinh \left(\pi \frac{a}{D}\right)}{x}\left[I_{i \frac{a}{D}}\left(\frac{R}{D}\right)\right]^{-2} \approx 2 \pi \frac{\left(1-e^{-\frac{2 \pi a}{D}}\right)}{\tau_{k}}
$$

and

$$
D_{e f f} \approx \frac{2 \pi^{2}}{\tau_{k}}\left(1+e^{-\frac{2 \pi a}{D}}\right),
$$

where $I_{i j}(x)$ is the modified Bessel function of first kind and $\tau_{k}$ is the time to escape over the potential barrier, that is the Kramers time $[17,18]$

$$
\tau_{k}=\tau_{0} e^{\frac{E}{D}}=\frac{2 \pi}{\sqrt{R^{2}-a^{2}}} e^{\frac{E}{D}}
$$

The height of the potential barrier $E$ for the harmonic function (9) is defined by the expression

$$
E=2 \sqrt{R^{2}-a^{2}}-2 a \arccos \frac{a}{R} .
$$

\section{Model functions}

In this section we propose two functions $W_{1}(x, t)$ and $W_{2}(x, t)$ as model functions for the time-dependent PDF, throughout the time scale $0 \leq t \leq \infty$ in the case of high potential barriers $E / D \gg 1$. As it was pointed out in reference [17], in this case there are two time scales in the Brownian motion. These are the intrawell relaxation time $\tau_{r}$ and the time to escape over the potential barrier $\tau_{k}$, that is the Kramers time. Our approach is based on two assumptions. First, the quasi-stationary distribution inside the potential well is established long time before an appreciable number of particles have escaped over the potential barrier, that is [17]

$$
\tau_{r} \ll \tau_{k} .
$$

Therefore, for high barriers $(E / D \gg 1)$ we can neglect the short relaxation time $\tau_{r}$ and consider the intrawell distribution in equation (4) as stationary not only for $t \rightarrow \infty$ but also starting from $t=0$. Secondly, the distribution of the interwell transitions (from a well to the neighboring wells) obeys the Gaussian coarse grained PDF at any time $0 \leq t \leq \infty$. This is our Gaussian approximation. The above assumptions lead to the same asymptotic distribution of equation (4), when $t \rightarrow \infty$, but the model distribution should be different from (4) for $t \rightarrow 0$. We can write the model distributions in the general form

$$
W_{i}(x, t)=W_{s t}(x) W_{G_{i}}(x, t),
$$

where $W_{s t}(x)$ is the periodically continued stationary intrawell distribution (7) and $W_{G_{i}}(x, t)$, with $i=1,2$ are the proposed Gaussian envelope model functions. In asymptotics $(t \rightarrow \infty)$, we should have

$$
W_{G_{i}}(x, t) \underset{t \rightarrow \infty}{\longrightarrow} \frac{e^{-\frac{\left(x-x_{0}-\nu t\right)^{2}}{4 D_{e f f}}}}{\sqrt{4 \pi D_{\text {eff }} t}} .
$$

When $t \rightarrow 0$, if initially the system was in an arbitrary point $x_{0}$ between two potential barriers, $2 \pi(k-1)<x_{0}<$ $2 \pi k$, we can consider that the stationary distribution inside the potential well near to the minimum at $x=m_{k}$ is set immediately. This property leads to the following condition

$$
W_{i}(x, t) \underset{t \rightarrow 0}{\longrightarrow} W_{s t}\left(x ; m_{k}\right) / 2 \pi,
$$

where $W_{s t}\left(x ; m_{k}\right)$ is the stationary distribution inside the $m_{k}$-th well. The stationary distribution is already included in (15). Then, in order to satisfy the condition (17), the Gaussian factor in equation (15) should tend to the uniform distribution $W_{0}(x)$ in the region between the two potential barriers $[2 \pi(k-1), 2 \pi k]$, when $t \rightarrow 0$. Therefore, by applying both assumptions we get the expression for the first model function $W_{G_{1}}(x, t)$ valid at any time $(0 \leq t \leq \infty)$

$$
\begin{gathered}
W_{G_{1}}(x, t)=\int_{-\infty}^{\infty} W_{0}\left(x_{0}\right) \frac{e^{-\frac{\left(x-x_{0}-\nu t\right)^{2}}{4 D_{\text {eff }} t}}}{4 \pi D_{\text {eff }} t} d x_{0} \\
=\frac{1}{2 \pi} \int_{2 \pi(k-1)}^{2 \pi k} \frac{e^{-\frac{\left(x-x_{0}-\nu t\right)^{2}}{4 D_{\text {eff }} t}}}{4 \pi D_{\text {eff }} t} d x_{0} \\
=\frac{1}{4 \pi}\left[\operatorname{Erf}\left(\frac{x-\nu t+2 \pi k}{2 \sqrt{D_{\text {eff }} t}}\right)-\operatorname{Erf}\left(\frac{(x-\nu t)+2 \pi(k-1)}{2 \sqrt{D_{\text {eff }} t}}\right)\right]
\end{gathered}
$$

where $\operatorname{Erf}(x)$ is the error function.

For high barriers $(E / D \gg 1)$, the stationary distribution is localized near the bottom of the potential well. Therefore, we can simplify the expression of $W_{s t}(x)$ given by equation (8). By Taylor expansion of the potential $u(x)$ around its $k$-th minimum $\left(m_{k}\right)$ and $k$-th maximum $\left(L_{k}\right)$, we get

$$
e^{\frac{ \pm u(x)}{D}} \approx e^{ \pm\left(\frac{u\left(x_{e x t r}\right)}{D}+\frac{u^{\prime \prime}\left(x_{e x t r}\right)}{2 D}\left(x-x_{e x t r}\right)^{2}\right)}
$$

where $x_{\text {extr }}=L_{k}, m_{k}$. The main contribution to the integral in equation (8) comes from a small neighborhood of the maximum $L_{k}$ next to the minimum $m_{k}$ (see Fig. 1). By using Taylor expansion (19) and taking into account that for the harmonic potential $u^{\prime \prime}\left(L_{k}\right)=-u^{\prime \prime}\left(m_{k}\right)$, we obtain 
the following stationary distribution in the $k$-th potential well $(2 \pi(k-1)<x<2 \pi k)$ for high barriers

$$
W_{s t}\left(x ; m_{k}\right)=\sqrt{\frac{2 \pi}{\sigma^{2}}} e^{-\frac{\left(x-m_{k}\right)^{2}}{2 \sigma^{2}}},
$$

where the variance of the distribution is $\sigma^{2}=D / u^{\prime \prime}\left(m_{k}\right)$. When $E / D \gg 1$, the tails of this distribution decrease so fast that in points where the potential is maximum, the distribution $(20)$ is almost zero $(\sigma \ll 2 \pi)$. The periodically continued stationary distribution will consist of Gaussian peaks (20) located in all the wells of the potential profile. Analytically it can be written as the following Fourier series [23]

$$
W_{s t}(x)=1+2 \sum_{n=1}^{\infty} e^{-\frac{n^{2}}{2} \sigma^{2}} \cos (n x) .
$$

The expressions of equations (18) and (21) define the first model distribution $W_{1}(x, t)$

$$
W_{1}(x, t)=W_{s t}(x) W_{G_{1}}(x, t) .
$$

It has a quite simple expression in comparison with that proposed in reference [15].

Nevertheless, to avoid the special function and make the expression (18) more simple, we propose another model distribution by replacing $W_{G_{1}}(x, t)$ with the following Gaussian function with two parameters $\beta_{1}$ and $\beta_{2}$

$$
W_{G_{2}}(x, t)=\frac{e^{-\frac{\left(x-m_{k}-\nu t\right)^{2}}{4 D_{e f f}\left(t+\beta_{2}\right)}}}{\sqrt{4 \pi D_{\text {eff }}\left(t+\beta_{1}\right)}} .
$$

This second Gaussian model distribution is an ansatz, which we will check in the next section. In order to satisfy condition (17) we should choose $\beta_{1}=\pi / D_{\text {eff }}$. Using the parameter $\beta_{2}$ we can make the function (23) closer to the uniform distribution (18) at $t=0$. From this viewpoint the parameter $\beta_{2}$ should satisfy the following condition $\sigma^{2} \ll 2 D_{\text {eff }} \beta_{2} \ll(4 \pi)^{2}$, which corresponds to the condition $E / D \gg 1$, when $\sigma^{2} \rightarrow 0$. Particularly, in the present paper we choose $\beta_{2}=\pi / 2 D_{\text {eff }}$ and further we consider the following second model function

$$
W_{2}(x, t)=W_{s t}(x) W_{G_{2}}(x, t),
$$

which does not include special functions. However, we should notice that the model function (24) has not correct normalization, when $\beta_{1} \neq \beta_{2}$. The normalization value is equal to 1 only for $t \rightarrow 0$ and $t \rightarrow \infty$, and it deviates from 1 for intermediate times $t \approx \beta_{i}$. Therefore, $W_{2}(x, t)$ is less adequate comparing to $W_{1}(x, t)$, but its form is simpler. Particularly, if we consider the harmonic periodic function (9) it reads

$$
W_{2}(x, t)=\left(1+2 \sum_{n=1}^{\infty} e^{-\frac{n^{2}}{2} \sigma^{2}} \cos (n x)\right) \frac{e^{-\frac{\left(x-m_{k}-\nu t\right)^{2}}{4 D_{e f f} t+2 \pi}}}{\sqrt{4 \pi D_{e f f} t+4 \pi^{2}}}
$$

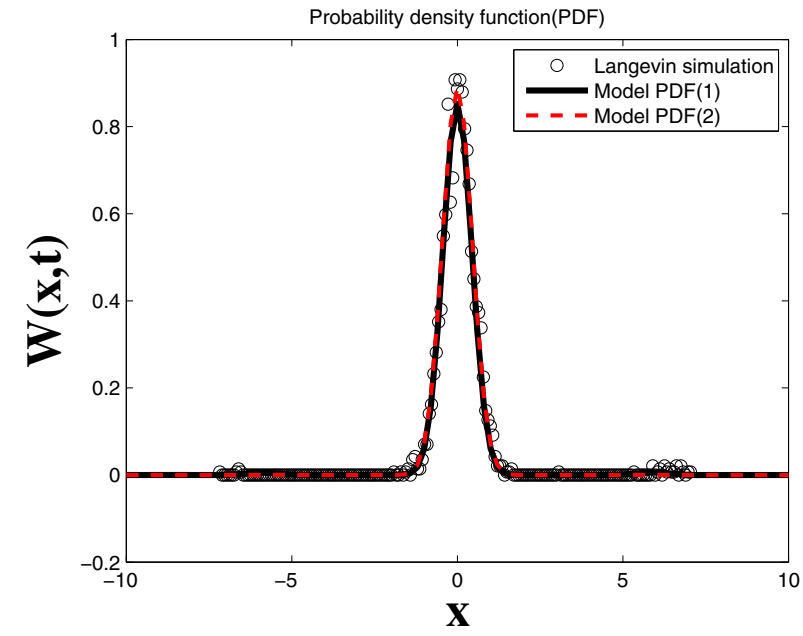

Fig. 3. (Color online) Model time-dependent PDFs, $W_{1}(x, t)$ (black tick line) and $W_{2}(x, t)$ (red dotted lines), compared with the time-dependent PDF obtained from numerical simulations of the Langevin equation (1) (black circles). Parameters: simulation time $t=0.01 \tau_{k}, E / D=10$. Notice the good agreement between the model PDFs and simulations.

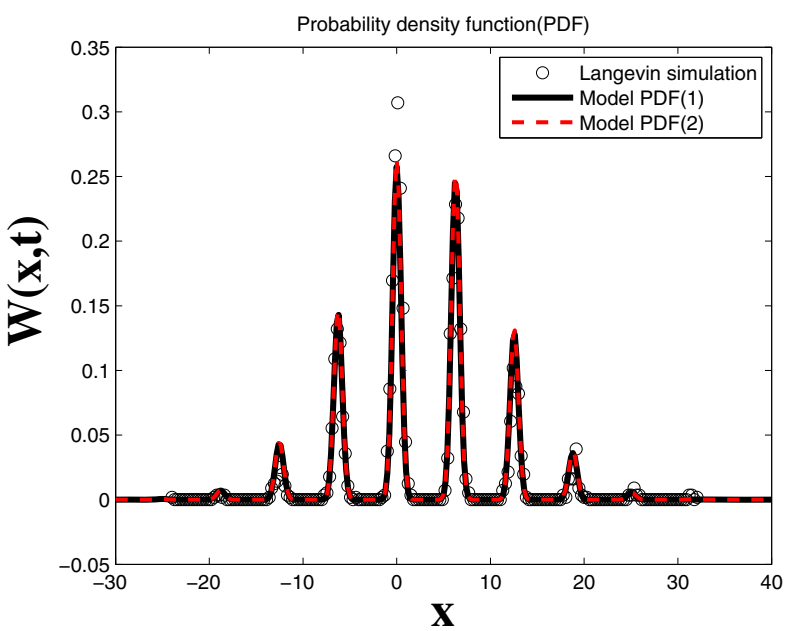

Fig. 4. (Color online) Model time-dependent PDFs, $W_{1}(x, t)$ (black tick line) and $W_{2}(x, t)$ (red dotted lines), compared with the time-dependent PDF obtained from numerical simulations of the Langevin equation (1) (black circles). Parameters: simulation time $t=\tau_{k}, E / D=10$.

\section{Results}

For the verification of the introduced model distributions $W_{1}(x, t)$ and $W_{2}(x, t)$, we compare them with the PDF obtained from numerical simulations of the Langevin equation (1). The numerical integration of equation (1) has been performed using the Runge-Kutta method of 4 -th order $[24,25]$. The PDFs shown in Figures $3-8$ have been obtained by calculating 200 values of $W(x, t)$, averaged over 2000 realizations for high barriers $(E / D=10)$ and on 20000 realizations for low barriers $E / D=3$. We consider harmonic periodical function (see Eq. (9)) with $R=10$ and slope $a=0.2$. Two noise intensity values are used, 


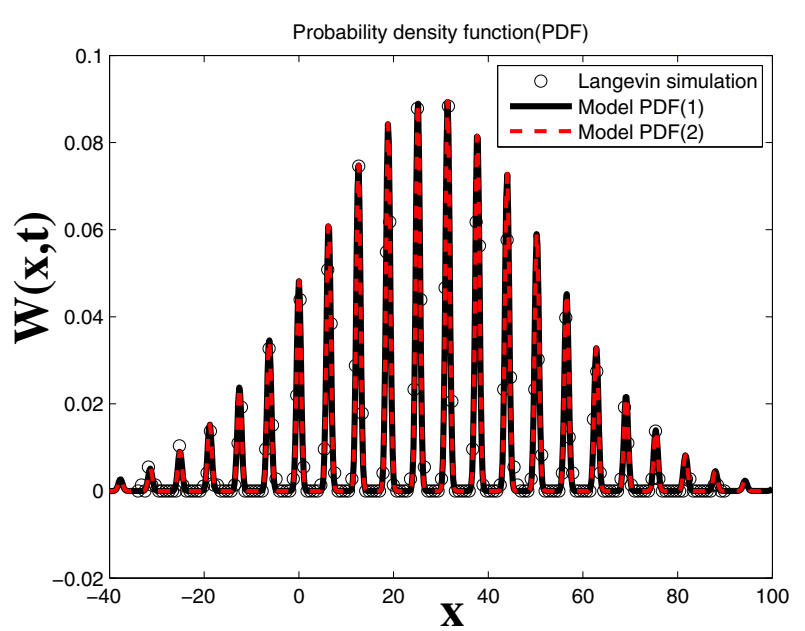

Fig. 5. (Color online) Model time-dependent PDFs, $W_{1}(x, t)$ (black tick line) and $W_{2}(x, t)$ (red dotted lines), compared with the time-dependent PDF obtained from numerical simulations of the Langevin equation (1) (black circles). Parameters: simulation time $t=10 \tau_{k}, E / D=10$.

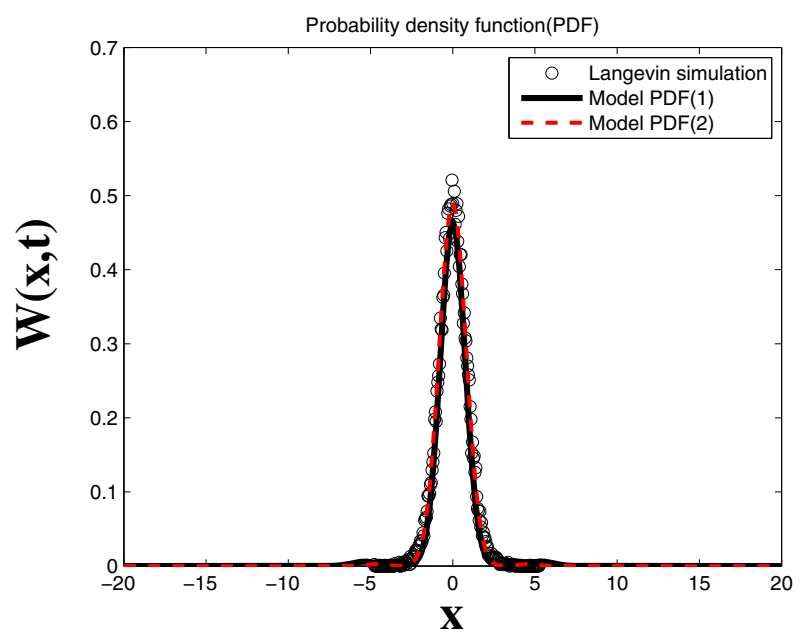

Fig. 6. (Color online) Model time-dependent PDFs, $W_{1}(x, t)$ (black tick line) and $W_{2}(x, t)$ (red dotted lines), compared with the time-dependent PDF obtained from numerical simulations of the Langevin equation (1) (black circles). Parameters: simulation time $t=0.01 \tau_{k}, E / D=3$.

namely $D=2$ and $D=6.6$, corresponding to the case of high and low barrier respectively.

The initial conditions for both cases are the same, that is $x_{0}=0$. This point is shifted from the minimum of the potential profile, which is located at the point $m_{1}=0.02$. The appropriate model distributions $W_{1}(x, t)$ (thick black line) and $W_{2}(x, t)$ (red dotted lines) together with the simulation results for the PDF (black circles) are shown in Figures 3-8.

First of all we note that there is no big difference between both the model distributions $W_{1}(x, t)$ and $W_{2}(x, t)$. The maximal difference is visible at $t=\tau_{k}$. The model distributions are close each other at $t=0.01 \tau_{k}$ and $t=10 \tau_{k}$, because at $t \rightarrow 0$ and $t \rightarrow \infty$ they coincide.

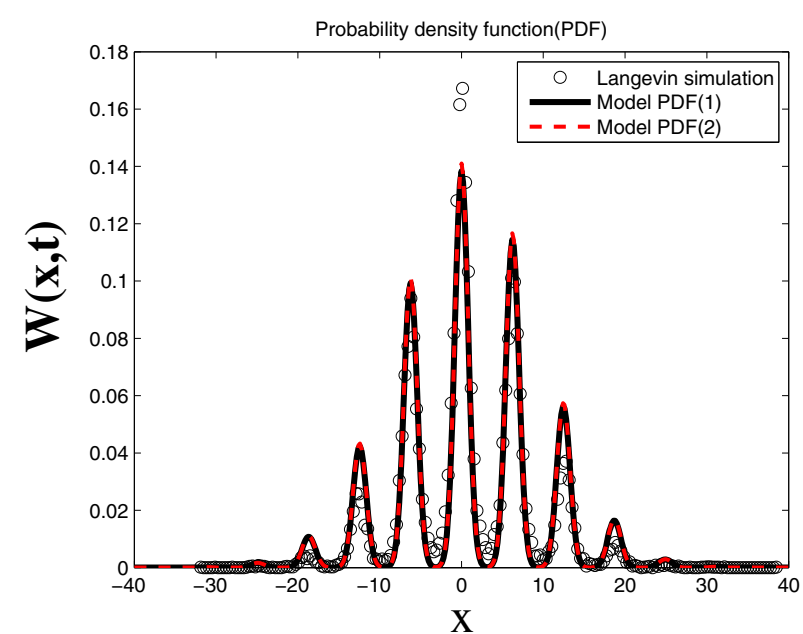

Fig. 7. (Color online) Model time-dependent PDFs, $W_{1}(x, t)$ (black tick line) and $W_{2}(x, t)$ (red dotted lines), timedependent PDF obtained from numerical simulations of the Langevin equation (1) (black circles). Parameters: simulation time $t=\tau_{k}, E / D=3$.

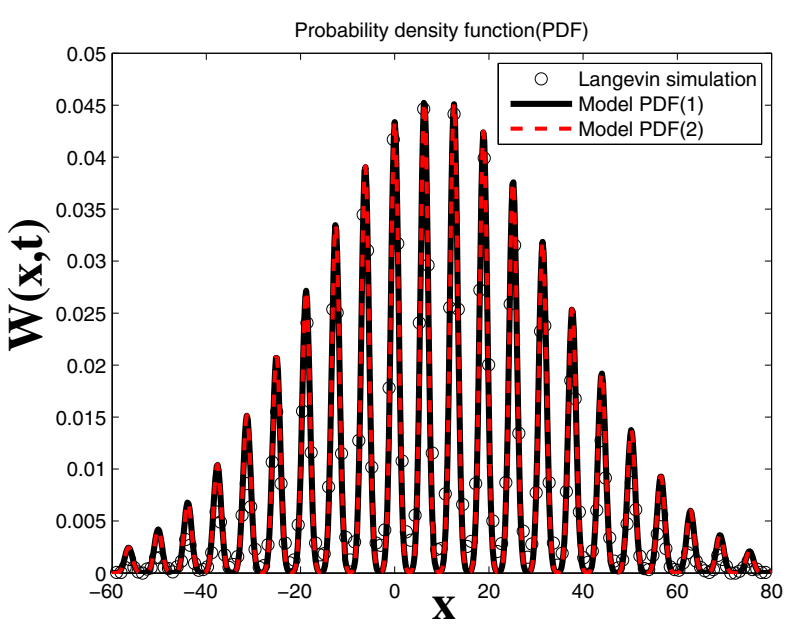

Fig. 8. (Color online) Model time-dependent PDFs, $W_{1}(x, t)$ (black tick line) and $W_{2}(x, t)$ (red dotted lines), compared with the time-dependent PDF obtained from numerical simulations of the Langevin equation (1) (black circles). Parameters: simulation time $t=10 \tau_{k}, E / D=3$.

As it was expected, the simulation results correspond better to the model PDFs, when the barriers are high $E / D=10$, because $W_{1}(x, t)$ and $W_{2}(x, t)$ are obtained for the case $E / D \gg 1$. When $E / D=3$, the differences between the model distributions and simulation results are slightly greater.

The most visible differences between models and simulations are also at $t=\tau_{k}$, the characteristic time of Brownian particles to cross the potential barrier. This is the time when the initial single-peak distribution starts to spread and some neighboring peaks arise. Both model distributions show a slightly wider spreading compared to the simulations, especially for the left diffusion, which is in the opposite direction with respect to the average drift. 


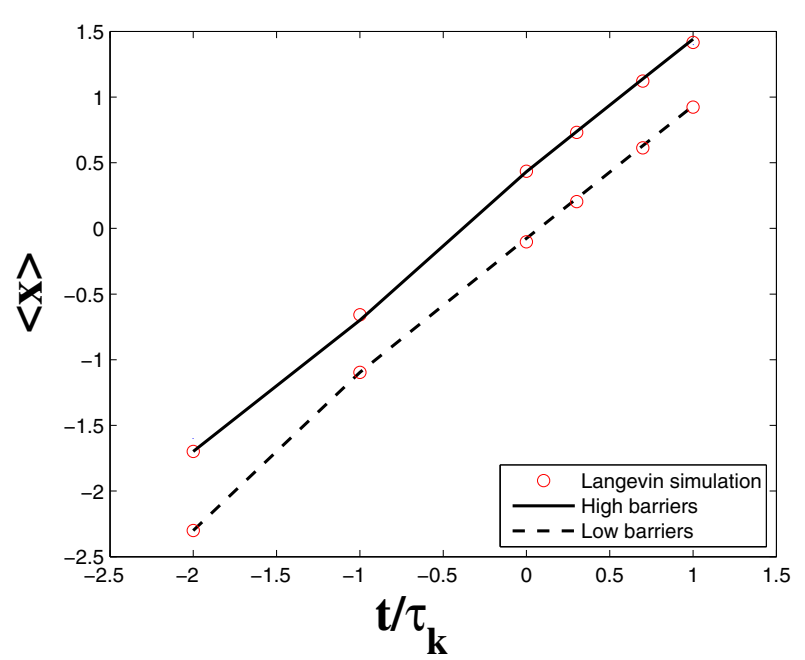

Fig. 9. (Color online) Comparison between the average drift values of the coordinate of the Brownian particle obtained by the second model PDF (25) and those obtained from simulation of the Langevin equation (1) (red circles), in the case of high $(E / D=10$, black line $)$ and low $(E / D=3$, black dashed line $)$ potential barriers. In both axes are reported the logarithms of the values.

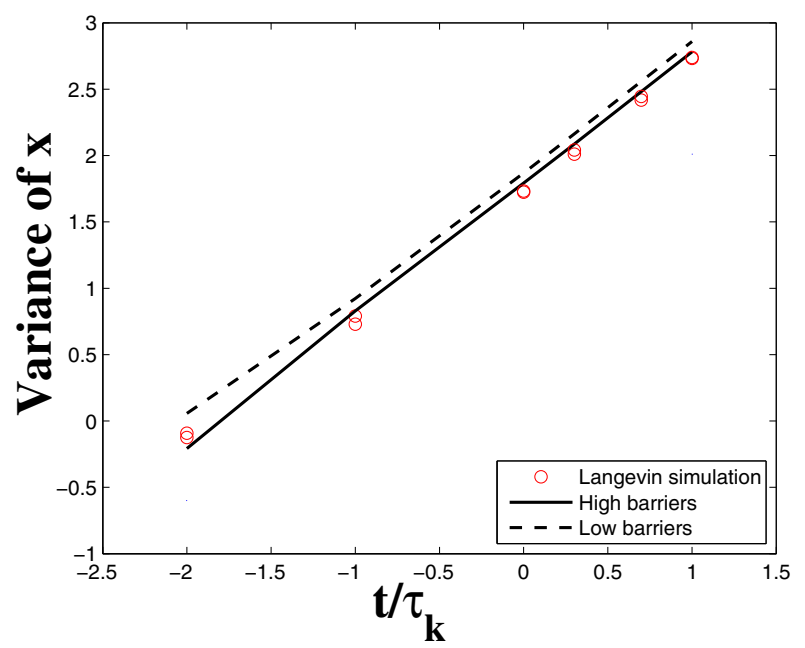

Fig. 10. (Color online) Comparison between the values of the variance of the Brownian particle coordinate obtained by the second model PDF (25) and those obtained from simulation of the Langevin equation (1) (red circles), in the case of high $(E / D=10$, black line) and low $(E / D=3$, black dashed line) potential barriers. In both axes are reported the logarithms of the values.

As a result, according to the normalization property, the main maximum of simulated PDF is higher than that of model distributions. However, this difference is very small, especially when $E / D=10$.

To further investigate this point we analyze the average drift of the Brownian particle coordinate and its variance. The black bold lines in Figures 9 and 10 show the average coordinate and its variance obtained from the second model PDF $(25)$ in the case of high $(E / D=10)$ and low $(E / D=3)$ potential barriers. The circles represent the same values calculated with numerical simulations of the
Langevin equation (1). The theoretical model results coincide with those obtained by simulation, at the time $t=\tau_{k}$ as well as for all other times. Concerning the variance, we can see that many points obtained from simulation of the Langevin equation lie slightly below the curves obtained from the second model. The difference is small and for the same curves obtained from the first model PDF, not reported here, is smaller. We can conclude that the small qualitative difference between the model PDFs and that obtained by simulations does not give rise to the considerable quantitative difference in the average drift of the particle coordinate and its variance. Finally, the numerical simulations of variance differ more when the ratio $E / D$ decreases, which is in accordance with the approximations used in this paper. This is also visible in Figures 3-8.

\section{Conclusions}

The proposed model expressions for the PDF of the Brownian particles diffusing in tilted periodical potentials have shown to be in a quite good agreement with the results obtained by numerical simulations in the case of high potential barriers, compared with the noise intensity. Particularly, we can conclude that with noise intensities smaller than $0.3 E$ both models describe well the actual non-stationary distribution of the Brownian particles.

The most relevant advantage of the presented model distributions is their simple form, so it can be used for different analytical applications such as the calculation of the spectrum for phase-locked loop systems.

In the present paper we have considered only harmonic function. This particular case of the periodical function leads to the Gaussian stationary intrawell distribution. In an arbitrary case the stationary distribution can be nonGaussian. However, the same approach for the model PDF construction can be used for an arbitrary form of a periodical functions as well.

Finally, we note that our analytical Gaussian approximations to the time-dependent probability density function in titled periodic potentials were derived in the overdamped regime. For smaller friction constants, inertial effects become important and a possible extension of this approach to the intermediate and low frequency regimes is not straightforward. This is because the dynamics is characterized by random transitions between the trapped state (the particle is trapped in one of the potential wells) and the running state (the particle moves along the potential profile without the trapping phenomenon) [1,4,5,26,27]. Only for moderately strong friction we expect that our approach could work. In the intermediate and low frequency regimes, due to the contribution of the running state, we expect non-Gaussian distributions with long tails. These underdamped dynamical regimes will be subject of our future investigations.

This work was supported by Russian Foundation for Basic Research (Project No. 08-02-01259). We acknowledge also support by the Italian Ministero dell'Istruzione, dell'Università e della Ricerca (MIUR). 


\section{References}

1. H. Risken, The Fokker-Planck Equation: Methods of Solution and Applications (Springer-Verlag, Berlin, 1984)

2. P. Reimann, Phys. Rep. 361, 57 (2002)

3. A.A. Dubkov, B. Spagnolo, Phys. Rev. E 72, 041104(8) (2005)

4. G. Costantini, F. Marchesoni, Europhys. Lett. 48, 491 (1999)

5. M. Borromeo, F. Marchesoni, Phys. Rev. Lett. 84, 203 (2000)

6. A.L. Pankratov, B. Spagnolo, Phys. Rev. Lett. 93, 177001 (2004)

7. G. Augello, D. Valenti, B. Spagnolo, Eur. Phys. J. B 78, $225(2010)$

8. V. Lindsey, Synchronization Systems in Communications and Control (Moscow, Sov. radio, 1978)

9. V.I. Tihonov, N.K. Kulman, Non-linear filtration and quasi-coherent signal receiving (Moscow, Sov. radio, 1975)

10. P. Reimann, C. Van den Broeck, H. Linke, P. Hänggi, J.M. Rubi, A. Perez-Madrid, Phys. Rev. E 65, 031104 (2002)

11. P. Hänggi, F. Marchesoni, Rev. Mod. Phys. 81, 387 (2009)

12. M.L. Dekhtyar, T.Ye. Korochova, V.M. Rozenbaum, Int. J. Quantum Chem. 110, 67 (2010)

13. R.L. Stratonovich, Topics in the Theory of Random Noise (Gordon and Breach Science Publishers Ltd, 1981), Vols. I and II
14. B. Lindner, M. Kostur, L. Schimansky-Geier, Fluct. Noise Lett. 1, R25 (2001)

15. R. Salgado-Garcia, F. Leyvraz, G. Martinez-Mekler, Phys. Rev. E 78, 061101 (2008)

16. A.N. Malakhov, Fluctuations in self-oscillatory systems (Moscow, Nauka, 1968)

17. H. Kramers, Physica, 7, 284 (1940)

18. P. Hänggi, P. Talkner, M. Borkovec, Rev. Mod. Phys. 62, 251 (1990)

19. A.N. Malakhov, Cumulative analysis of random nongaussian processes and their transformations (Moscow: Sov. radio, 1978)

20. W. Gardiner, Handbook of stochastic methods (Springer, Berlin, 1990)

21. N.V. Agudov, A.V. Safonov, Fluct. Noise Lett. 5, L283 (2005)

22. P. Reimann, Phys. Rev. Lett. 86, 4992 (2001)

23. G.P. Tolstov, Fourier series (Publications, New York, London, 1976)

24. N.N. Nikitin, S.V. Pervachev, V.D. Razevig, Avtomatika and Telemehanica (in Russian) 4, 133 (1975)

25. P.E. Kloeden, E. Platen, Numerical Solution of Stochastic Differential Equations (Springer-Verlag, Berlin, 1999)

26. A. Barone, G. Paternò, Physics and Application of the Josephson Effect (Wiley, New York, 1982)

27. A.I. Shushin, Chem. Phys. 370, 244 (2010) 